\title{
ON THE BELTRAMI EQUATION, ONCE AGAIN: 54 YEARS LATER
}

\author{
Bogdan Bojarski \\ Instytut Matematyczny Polskiej Akademii Nauk \\ ul. Sniadeckich 8, 00-956 Warszawa, Poland; B.Bojarski@impan.gov.pl
}

\begin{abstract}
We prove that the quasiregular mappings given by the (normalized) principal solution of the linear Beltrami equation (1) and the principal solution of the quasilinear Beltrami equation are inverse to each other. This basic fact is deduced from the Liouville theorem for generalized analytic functions. It essentially simplifies the known proofs of the "measurable Riemann mapping theorem" and its holomorphic dependence on parameters.
\end{abstract}

The first global, i.e. defined in the full complex plane $\mathbf{C}$ and expressed by an explicit analytical formula, solution of the Beltrami equation

$$
w_{\bar{z}}-q(z) w_{z}=0
$$

was given by Vekua in the years 1953-54 and it appeared in the first issue of Doklady for 1955 [32].

Vekua in [32] considered the equation (1) with compactly supported $q(z), q(z) \equiv 0$ for $|z|>R$, for some finite $R$, satisfying the uniform ellipticity condition

$$
|q(z)| \leq q_{0}<1, \quad q_{0}-\text { const. }
$$

In [32] he considers the class of solutions of (1) represented by the Cauchy complex potential $T \omega$ in the form

$$
w(z)=-\frac{1}{\pi} \int_{C} \frac{\omega(\zeta) d \sigma_{\zeta}}{\zeta-z}+\phi(z) \equiv T \omega+\phi(z)
$$

where $\omega(\zeta)$ is a complex density, $\omega \in L^{p}(\mathbf{C}), p>1$, and $\phi(z)$ is an entire holomorphic function.

The function $w=w(z)$ is a $W_{\text {loc }}^{1, p}(\mathbf{C})$ solution of (1) iff the density $\omega$ is a solution of the singular integral equation

$$
\omega-q(z) S \omega=q(z) \phi^{\prime}(z) \equiv h(z),
$$

with the singular integral

$$
S \omega=-\frac{1}{\pi} \int_{C} \frac{\omega(\zeta)}{(\zeta-z)^{2}} d \sigma_{\zeta}
$$

understood in the sense of the Cauchy principal value.

It was probably Vekua who first introduced the singular integral operator $S$ to the study of elliptic equations in the plane. It appeared as early as 1952-53 in connection with the study of general boundary value problems, specifically the

doi:10.5186/aasfm.2010.3504

2000 Mathematics Subject Classification: Primary 30C62; Secondary 35J55.

Key words: Beltrami operators, quasiconformal mappings, elliptic PDE's.

This paper was partially supported by Grant 1-P03A-008-29, MEN, and by EU Marie Curie programmes CODY and SPADE2. 
Poincaré boundary value problem, in the theory of generalized analytic functions (GAF - for short), which was defined and developed in Vekua's famous paper [30]. See also [31] and the Ph.D. dissertation [4], [5], prepared in 1953-54.

Later the operator $S \omega$ was called the Hilbert transform.

The main role of the operator $S$ in the Vekua school was to transform the derivative $w_{\bar{z}}$ into $w_{z}$ for compactly supported smooth functions $w \in C_{0}^{\infty}(\mathbf{C})$,

$$
S\left(w_{\bar{z}}\right)=w_{z}=\frac{\partial}{\partial z} T\left(w_{\bar{z}}\right) .
$$

Since for $w \in C_{0}^{\infty}(\mathbf{C})$ the entire function $\phi(z)$ in (3) reduces to $\phi \equiv 0$, integration by parts then gives $\left\|w_{\bar{z}}\right\|_{L^{2}}=\left\|w_{z}\right\|_{L^{2}}$ and

$$
\|S \omega\|_{L^{2}}=\|\omega\|_{L^{2}}
$$

where $\|\cdot\|_{L^{2}}$ denotes the $L^{2}$ norm of square integrable functions. In consequence $S$ extends as a unitary isometry to the Hilbert space $L^{2}(\mathbf{C})$.

For further reference we also note the equivalent description in terms of the Fourier transform

$$
\widehat{S \omega}(\xi)=\frac{\xi}{\bar{\xi}} \widehat{\omega}(\xi) \quad \text { for every } \quad \xi \in \mathbf{C} \backslash\{0\} .
$$

The uniform ellipticity condition (2) gives the $L^{2}$ norm estimate for the operator $q S$,

$$
\|q S\|_{L^{2}} \leq q_{0}<1
$$

and immediately leads to the unconditional solvability of the integral equation (4) in the space $L^{2}(\mathbf{C})$ by the Neumann series or successive approximations.

Lemma 1. For arbitrary measurable dilatation $q(z)^{1}$, satisfying (2), the integral equation (4) has a unique solution in $L^{2}(\mathbf{C})$ given by the formula

$$
\omega=(1-q S)^{-1} h .
$$

This means that the differential Beltrami equation (1) with the compactly supported coefficient $q=q(z)$ has a unique solution in the Sobolev space $W_{\mathrm{loc}}^{1,2}(\mathbf{C})$, admitting a holomorphic extension of the form (3) outside the support of $q$.

Actually, by [31], any generalized (weak) solution of (1) in the space $W_{\text {loc }}^{1,2}(\mathbf{C})$, can be obtained by the described process. However, below, for the purposes of the theory of quasiconformal mappings, we are interested in very special solutions of (1) only.

As a convolution type operator $S$ commutes with differential operators. Moreover, it preserves the Hölder-Zygmund classes $C^{k, \alpha}$ with $0<\alpha<1$ and $C^{\infty}(\mathbf{C}) \cap$ $L^{2}(\mathbf{C})$. In particular, this implies that for $q(z)$ and $h C^{\infty}$-smooth, or of the class $C^{k, \alpha}$, the uniquely determined $L^{2}$ solutions of the integral equation (4) are as smooth as the data $q$ and $h$ allow. We formulate this as

Lemma 2. For compactly supported $C^{\infty}$-smooth dilatation $q(z)$ the weak $W_{\text {loc }}^{1,2}$ solutions of the Beltrami equation (1) are $C^{\infty}$-smooth.

\footnotetext{
${ }^{1}$ In the classical geometric theory of $K$-quasiconformal mappings ( $K$-QC mappings) the coefficient $q(z)$ of the Beltrami equation (1) is called the complex dilatation of the mapping, the constant $q_{0}$ in (2) is denoted by $k$ and is related to $K$ by the formula $k=\frac{K-1}{K+1}$. We will comment on the geometric meaning of the constants $k$ and $K$ later, though below we freely use the terminology of the theory of QC mappings.
} 
The proof of Lemma 2 is rather direct, relying on the classical tools of standard potential theory and is described in detail in Vekua's book [33].

By the Calderón-Zygmund theorem [15] the operator $S$ acts also as a bounded operator in $L^{p}(\mathbf{C})$ for each $p, 1<p<\infty$, and its norm $\mathscr{A}_{p}$ is continuous at $p=2$. Thus

$$
\mathscr{A}_{p} \cdot q_{0}<1 \text { for } 2 \leq p<2+\varepsilon
$$

and the equation $\omega-q S \omega=h$ is uniquely solvable:

$$
\omega=(I-q S)^{-1} h, \quad \omega \in L^{p},
$$

for any $h \in L^{p}$ and $p$ satisfying (10), what we henceforth assume. In particular, for any measurable dilatation $q(z)$ the $L^{2}$ solution $\omega$ of equation (4) is actually in some $L^{p}, p>2$. Thus, in other words, the $W_{\text {loc }}^{1,2}$ solutions of (1) belong to $W_{\text {loc }}^{1, p}, p>2$. In particular, they are continuous ( $\alpha$-Hölder, $\left.\alpha=1-\frac{2}{p}>0\right)$.

For $\phi(z) \equiv z, h(z) \equiv q(z)$, formula (3) gives a particular solution of the Beltrami equation (1)

$$
w(z) \equiv z-\frac{1}{\pi} \int_{\mathbf{C}} \frac{\omega(\zeta)}{\zeta-z} d \sigma_{\zeta}
$$

where $\omega$ is the unique solution of the equation

$$
\omega-q(z) S \omega=q(z) .
$$

Following Vekua [32], see also [6], we call (12) the principal solution of the Beltrami equation.

A fundamental issue of the theory of elliptic equations and planar quasiconformal mappings was the understanding that the formulae (12)-(13) give a univalent solution of the uniformly elliptic Beltrami equation (1)-(2) realizing a homeomorphic quasiconformal mapping of the complex plane with the assigned measurable complex dilatation $q(z)$ (the Measurable Riemann Mapping Theorem). This was achieved in 1954, and published in the first months of 1955 in [6], [7], [32], by the collaborative efforts of Vekua and the author.

Let us briefly recall the main steps. The existence of $W_{\mathrm{loc}}^{1,2}(\mathbf{C})$ solutions was clear from the outset and the problem essentially reduced to the $L^{2}$ isometry of the operator $S$ and the classical properties of the complex potential $T: L^{2} \rightarrow W^{1,2}$, described in [30], [31]. The idea of applying the Calderón-Zygmund theorem [15] and, thus, extending the range of admissible parameters $p$ to the interval $2-\varepsilon<p<2+\varepsilon$ for some positive $\varepsilon$, due to the author [6], [33], immediately allowed us to consider $W_{\text {loc }}^{1, p}$ solutions, $p>2$, and, by the Sobolev imbedding theorems, or classical properties of the complex potentials $T \omega, \alpha$-Hölder continuous solutions with $\alpha=1-\frac{2}{p}>0$.

This we formulate in

Proposition 1. The Beltrami equation (1) with an arbitrary measurable dilatation $q(z)$, satisfying (2) and compactly supported, always admits the solution of the form (12) in the Sobolev class $W_{\text {loc }}^{1, p}, p>2$. Moreover, the norms $\left\|w_{z}-1\right\|_{L_{p}},\left\|w_{\bar{z}}\right\|_{L_{p}}$ of this solution are uniformly bounded by quantities depending only on $q_{0}$ in (2) and $\|q\|_{L_{p}}$ (or the support of $\left.|q|\right)$.

Not necessarily homeomorphic solutions of the Beltrami equations are known as quasiregular mappings. By formulas (3) and (4) above they are relatively easy to construct. The proof that univalent solutions exist at all, the more so, that the 
solutions (12) are homeomorphisms onto, is much more subtle. In the papers [6], [32] it splits into

Proposition 2. If the dilatation $q(z)$ is sufficiently smooth, then the mapping (12) is a homeomorphism onto, i.e., it is a quasiconformal mapping of the complex plane.

and

Proposition 3. For arbitrary measurable dilatation $q(z)$, satisfying condition (2), the formulae (12)-(13) realize a quasiconformal mapping of the complex plane with the assigned dilatation almost everywhere.

In the context of papers [6], [32], Proposition 2 was proved by Vekua in [32] for the class of Hölder continuous dilatations, though for the purposes of [6], where the first complete proof of Proposition 3 was given, it is enough to have Proposition 2 for dilatations of much higher regularity, say of class $C^{\infty}$, only.

In [32] a local version of Proposition 2 is proved first (Proposition 4 below). The global version of Proposition 2 is obtained by some general, global, geometric monodromy type argument recalled below.

The idea of the present paper is to prove Proposition 2 without appealing to Proposition 4 , but by direct construction of a quasiregular mapping, i.e. a $W_{\text {loc }}^{1, p}$, $p>2$, solution of some other Beltrami equation, a quasilinear one, and such that the constructed mapping is actually the two-sided inverse to the mapping given by formula (12).

The proof of the implication Proposition $2 \Longrightarrow$ Proposition 3 proceeds as in $[6]$ (it was repeated in $[8]^{2},[33]$ ).

In view of the approximating procedure described in [6] it is, obviously, enough to consider the Beltrami equation (1) with dilatation $q(z)$ of arbitrary high smoothness (even $C^{\infty}$ ).

To this aim we consider, parallel to equation (1), the quasilinear equation for the mappings $z=z(w)$ of the image plane $C_{w}$ in (1) to the source plane $C_{z}$

$$
\frac{\partial z}{\partial \bar{w}}+q(z) \frac{\overline{\partial z}}{\partial w}=0
$$

We call it the conjugate (quasilinear) Beltrami equation.

Now we are interested in a particular solution of (14) of the form

$$
\psi(w)=w+T \widetilde{\omega} \equiv w-\frac{1}{\pi} \int_{\mathbf{C}} \frac{\widetilde{\omega}(\zeta)}{\zeta-w} d \sigma_{\zeta}
$$

with $\widetilde{\omega} \in L^{p}$, for some $p>2$.

(15) is a solution of (14) of the Sobolev class $W_{\text {loc }}^{1, p}\left(\mathbf{C}_{w}\right)$ iff the complex density $\widetilde{\omega}$ is a solution of the singular integral equation

$$
\widetilde{\omega}+\widetilde{q}(w) \bar{S} \widetilde{\omega}=-\widetilde{q}(w)
$$

with $\widetilde{q}(w) \equiv q(\psi(w))$. Hence $\psi(w)-w$ is in the class $W^{1, p}$ and $\psi(w)$ is the solution of the conjugate Beltrami equation

$$
\frac{\partial \psi}{\partial \bar{w}}+\widetilde{q}(w) \frac{\overline{\partial \psi}}{\partial w}=0
$$

\footnotetext{
${ }^{2}$ English translation of [8] has been recently edited as Report 118 of the Jyväskylä University.
} 
with $\widetilde{q}(w)$ at least Hölder continuous with exponent $\alpha=1-\frac{2}{p}>0$. In the terminology adopted above the mapping $\psi(w)$ is a quasiregular mapping of the complex plane $\mathbf{C}_{w}$ into the plane $\mathbf{C}_{z}$.

Considered as an operator equation for the unknown density $\widetilde{\omega}(w),(16)$ is a highly nonlinear operator equation. However, its solvability in $L^{p}$ spaces is easily controlled.

Lemma 3. The quasilinear conjugate Beltrami equation with smooth dilatation $q(z)$ always admits a solution of type (15) in some $W_{\mathrm{loc}}^{1, p}, p>2$. Equivalently, the nonlinear equation (16) always admits a solution $\widetilde{\omega}$ in $L^{p}\left(\mathbf{C}_{w}\right)$ (compactly supported) for some $p>2$.

The solution (15) of (14) is unique. We will comment on the proof of Lemma 3 later. Here we remark only that in [6], [7] and [8] there is a plentiful of theorems of the type of Lemma 3 and their proofs are constructed along, the, now standard, procedure based on Banach or Leray-Schauder fixed point theorems and a priori estimates directly deduced from the linear uniformly elliptic equations (4) and (16) written in the form

$$
\widetilde{\omega}+\widetilde{q} \bar{S} \widetilde{\omega}=h, \quad h \in L^{p} .
$$

For our proof of Proposition 2 we shall need the Liouville theorem for generalized analytic functions (GAF) of Vekua, introduced in [30], and in his earlier works and thoroughly described in [8] and [33].

Lemma 4. Let $w=w(z)$, in $W^{1,2}(\mathbf{C})$, be a (generalized) solution of the equation

$$
w_{\bar{z}}-q(z) w_{z}=A w
$$

with the coefficient $q(z)$ : measurable, compactly supported and satisfying uniform ellipticity condition (2), and $A \in L^{p}(\mathbf{C})$ for some $p>2$. For simplicity assume also that $A$ is compactly supported. If $w$ vanishes at $\infty$, i.e. $|z||w(z)|<C$ for all $z$, then $w \equiv 0$.

Proof. If $A \equiv 0$ the equation (19) reduces to the Beltrami equation (1) and then, denoting $w_{\bar{z}}=\omega \in L^{2}(\mathbf{C})$, by the classical result of Vekua [32], the solution $w(z)$ may be represented as the complex potential $w(z) \equiv T(\omega)(\phi(z) \equiv 0$ in $(3))$. Now (19) implies that the compactly supported density $\omega$ is a solution of $\omega-q S \omega \equiv 0$, hence $\omega \equiv 0$ by $L^{2}$ isometry of the operator $S$. Consequently $w \equiv 0$.

If $A \in L^{p}(\mathbf{C})$, consider the solution $\widetilde{\omega}$ of the integral equation

$$
\widetilde{\omega}-q S \widetilde{\omega}=A .
$$

Then $\operatorname{supp} \widetilde{\omega} \subset(\operatorname{supp} q \cup \operatorname{supp} A)$ and the substitution

$$
w(z)=f(z) e^{\varphi(z)}, \quad \varphi(z) \equiv T(\widetilde{\omega}),
$$

reduces the lemma to the Beltrami equation for $f(z)$. The assumption $p>2$ for $A$ allows to obtain $\widetilde{\omega} \in L^{p^{\prime}}$, for some $p^{\prime}>2$, possibly less than $p$, but sufficient to keep $\varphi(z)$ uniformly Hölder continuous and bounded. This ends the sketch of the proof, for details see [8] and [33].

Corollary 1. The conclusion of Lemma 4 holds also for mappings $w=w(z)$ in $W^{1,2}(\mathbf{C}), w(\infty)=0$, satisfying the inequality

$$
\left|w_{\bar{z}}-q_{1}(z) w_{z}-q_{2}(z) \overline{w_{z}}\right| \leq A(z)|w(z)|
$$


if the coefficients $q_{1}, q_{2}$ have compact support and satisfy the uniform ellipticity condition

$$
\left|q_{1}(z)\right|+\left|q_{2}(z)\right| \leq q_{0}<1, \quad q_{0} \text { - const. }
$$

and $A \in L^{p}(\mathbf{C}), p>2$, vanishes for $|z|$ big enough.

Lemma 4, the Corollary and the proof above, are given here only for the completeness of the presentation. They could be simply referred to Theorems 4.1 and 4.2 and the remark in Sections 4.1 and 4.2 in [8].

The important concept of GAF, corresponding to the system (19), and discussed in [30] and [33] under the term: generalized constants (or generalized units), is also useful in the global theory of the Beltrami equation (1).

Lemma 5. In the conditions of Lemma 4 the equation (19) has a unique solution defined in the full complex plane $v=v(z), z \in \mathbf{C}$, regular at $z \rightarrow \infty$, and such that $v(\infty)=1$. This solution does not vanish for any $z \in \mathbf{C}$,

$$
v(z) \neq 0 \text {. }
$$

Proof. The required solution is constructed by the formula

$$
v(z)=e^{\varphi(z)}, \quad \varphi(z) \equiv T(\omega),
$$

where $\omega$ is the solution of the singular integral equation (20). Uniqueness reduces to the (obvious) Liouville theorem for the Beltrami equation (1) and follows also from Lemma 4.

Lemma 5, as Lemma 4 above, could be also referred to [8].

Corollary 2. The derivative $w_{z}$ of the principal solution (12) of the Beltrami equation with smooth dilatation $q(z)\left(q(z) \in W^{1, p}, p>2\right.$, is enough) is a generalized constant for equation (19). In particular,

$$
w_{z} \equiv 1+S \omega \neq 0 \quad \text { for all } \quad z \in \mathbf{C} .
$$

Proof. Putting $u=w_{z}$ and differentiating the Beltrami equation (1) with respect to $\partial_{z}$ we obtain

$$
u_{\bar{z}}-q(z) u_{z}=A u, \quad A \equiv q_{z} .
$$

Moreover, $u(z)=1+S \omega \rightarrow 1$ for $|z| \rightarrow \infty$, hence, by Lemma 4 , the conclusion.

The above proof is taken from [11].

Corollary 2 immediately implies the following

Proposition 4. In the conditions of Proposition 2 the principal (quasiregular) solution (12) is a local homeomorphism.

Proof. Indeed, the Jacobian

$$
J_{w}=\left|w_{z}\right|^{2}-\left|w_{\bar{z}}\right|^{2}=\left|w_{z}\right|^{2}(1-|q(z)|)^{2} \neq 0
$$

for every $z \in \mathbf{C}$.

In [32] Vekua deduced Proposition 2 from Proposition 4 by appealing to the "argument principle" for local homeomorphisms of the complex plane. It was also well known that the monodromy theorem for open mappings of the Riemann sphere $S^{2}$ or the closed plane $\widehat{\mathbf{C}}$ may also be used to deduce Proposition 2 from Proposition 4 . Even reference to the famous "uniformization" theorem was exploited sometimes! 
Though all the above statements are "well known", "well understood" and "intuitively obvious" for geometers and practicing complex analysts, neither of them can be considered "elementary".

Lemma 6 and Proposition 5 below reduce the proof of Proposition 2 to the Liouville theorem for Vekua's generalized analytic functions in the extended complex plane $\widehat{\mathbf{C}}$ : our Lemma 4 and Corollary 1 .

Let us now consider the Beltrami equation (1) with a smooth compactly supported dilatation $q(z)$ and the conjugate Beltrami quasilinear equation (14).

Lemma 6. Let $\chi=\chi(z)$ be the normalized (principal) solution (12) of equation (1) and $\psi=\psi(w)$ the principal solution (15) of the quasilinear equation (14). Consider the composed mappings

$$
\begin{aligned}
\widetilde{\phi}(w) & =\chi \circ \psi(w), & & \widetilde{\phi}: \mathbf{C}_{w} \rightarrow \mathbf{C}_{w}, \\
\phi(z) & =\psi \circ \chi(z), & & \phi: \mathbf{C}_{z} \rightarrow \mathbf{C}_{z} .
\end{aligned}
$$

Then $\widetilde{\phi}=\widetilde{\phi}(w)$ is a solution of the Cauchy-Riemann equation

$$
\frac{\partial \widetilde{\phi}}{\partial \bar{w}}=0
$$

and $\phi(z)$ satisfies the inequality

$$
\left|\phi_{\bar{z}}-\widetilde{q}(z)\left(\phi_{z}-\bar{\phi}_{z}\right)\right| \leq A(z)|\phi(z)-z|
$$

with a bounded, compactly supported function $A(z)$ and

$$
\widetilde{q}(z) \equiv \frac{q(z)}{1+|q(z)|^{2}}
$$

Proof. (25) is immediate:

$$
\frac{\partial \widetilde{\phi}}{\partial \bar{w}}=\frac{\partial \chi}{\partial z} \frac{\partial \psi}{\partial \bar{w}}+\frac{\partial \chi}{\partial \bar{z}} \frac{\partial \bar{\psi}}{\partial \bar{w}}=\frac{\partial \chi}{\partial z}\left(\frac{\partial \psi}{\partial \bar{w}}+q(\psi(w)) \frac{\overline{\partial \psi}}{\partial w}\right)=0
$$

The calculations for (26) are somewhat more complicated. We have, keeping in mind $(24)$ and $w=\chi(z)$,

$$
\begin{aligned}
& \phi_{\bar{z}}=\psi_{w} \chi_{\bar{z}}+\psi_{\bar{w}} \overline{\chi_{z}}=\psi_{w} q(z) \chi_{z}-q(\psi(\chi)) \overline{\psi_{w}} \overline{\chi_{z}} \\
& =q(z)\left[\psi_{w} \chi_{z}-\overline{\psi_{w}} \overline{\chi_{z}}\right]-[q(\phi(z))-q(z)] \overline{\psi_{w}} \overline{\chi_{z}}, \\
& \phi_{z}=\psi_{w} \chi_{z}+\psi_{\bar{w}} \overline{\chi_{\bar{z}}}=\psi_{w} \chi_{z}-q(\phi(z)) \overline{q(z)} \overline{\chi_{z}} \overline{\psi_{w}} \\
& =\psi_{w} \chi_{z}-|q(z)|^{2} \overline{\chi_{z}} \overline{\psi_{w}}+[q(z)-q(\phi(z))] \overline{q(z)} \overline{\psi_{w}} \overline{\chi_{z}},
\end{aligned}
$$

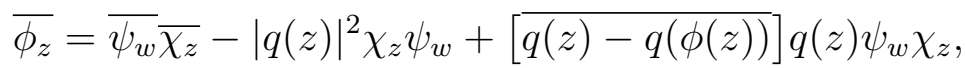

$$
\begin{aligned}
& \phi_{z}-\overline{\phi_{z}}=\left(1+|q(z)|^{2}\right)\left(\psi_{w} \chi_{z}-\overline{\psi_{w}} \overline{\chi_{z}}\right)+\text { terms linear in }[q(z)-q(\phi(z))] \text {, }
\end{aligned}
$$

and we see that (26) holds with some explicit expression for $A(z)$ in terms of the quantities involved. Since $q, q(\phi(z))$ are at least $C^{1}$ smooth, thus Lipschitz, and compactly supported and $\psi(w)$ and $\chi$ are at least $C^{1}$ smooth also, $A(z)$ satisfies all the required properties. Also, $|\widetilde{q}(z)| \leq \widetilde{q}_{0}<\frac{1}{2}$.

Lemma 6 and its proof have a nice geometric interpretation in terms of Lavrentiev fields (characteristics), see below, p. 70, and also [12] and [34], [27]. 
Lemmas 4 and 6 lead to Proposition 2 with a completely new direct proof. We formulate it as

Proposition 5. The normalized solutions (12) and (15) of the smooth Beltrami equation and the conjugate quasilinear equation are homeomorphisms of the complex planes $\mathbf{C}_{z} \leftrightarrow \mathbf{C}_{w}$ inverse to each other, i.e. the formulas hold

$$
\chi \circ \psi(w) \equiv w \quad \text { and } \quad \psi \circ \chi(z) \equiv z .
$$

Proof. Indeed, by Lemma $6 \widetilde{\phi}(w)$ is holomorphic in $w$ and obviously $\sim w$ at infinity, hence $\widetilde{\phi}(w) \equiv w$. If we consider $W(z)=\phi(z)-z$ then in view of $(26)$

$$
\left|W_{\bar{z}}-\widetilde{q}(z)\left(W_{z}-\bar{W}_{z}\right)\right| \leq A(z)|W|, \quad W(\infty)=0,
$$

and, by Corollary $1, W \equiv 0$. Thus both formulas (27) are proved. Other statements of the proposition are direct consequences of (27).

We also have an important corollary.

\section{Corollary 3.}

$$
J_{\chi} \cdot J_{\psi} \equiv 1
$$

where $J_{\chi}=\left|\chi_{z}\right|^{2}-\left|\chi_{\bar{z}}\right|^{2}$ and $J_{\psi}=\left|\psi_{w}\right|^{2}-\left|\psi_{\bar{w}}\right|^{2}$ are the Jacobians. In particular,

$$
J_{\chi} \neq 0 \quad \text { and } \quad J_{\psi} \neq 0
$$

at every point. Actually $J_{\chi} \geq c_{\chi}>0$ and $J_{\psi} \geq c_{\psi}>0$ for positive constants (in general, dependent on the mapping).

(29) is also a direct consequence of Lemma 5 above.

In the foundational study of Vekua and the author on Beltrami equations described in [32], [33] and [6], [8] formulas of the type (27) and (15) play an important role. However there, given $\chi=\chi(z)$ defined by (12), for a sufficiently smooth dilatation $q(z)$, it is first proved (Proposition 2) that $\chi=\chi(z)$ is a homeomorphism and the formulas $(27)$ are used to define $\psi=\psi(w)$. Only in the next step $\psi(w)$ is shown to be a solution of the conjugate Beltrami quasilinear equation (14) which may be represented in the form (15).

These facts are the cornerstones of the theory of Beltrami equations in the plane as developed and described in detail in [32] and [6]. In [6], [8] there are also established the basic a priori estimates in the $L^{p}$ and $W^{1, p}$ norms, for $2<p<2+\varepsilon$, for normalized solutions of the Beltrami equation (1) and their inverses $\psi=\psi(w)$ in terms of the dilatation $q(z)$. These estimates are preserved under various limiting processes, $q_{n} \rightarrow q$ for $n \rightarrow \infty$, even in the space of bounded measurable functions with topology defined by almost everywhere convergence as long as the uniform ellipticity condition (2) is uniformly fulfilled: $\sup _{z, n}\left|q_{n}\right| \leq q_{0}<1$. For the proofs of all these facts and their important consequences we refer to [6]. See also [8] and [33].

After 1955-57, most of the numerous publications (and all monographs) on the generalized Riemann mapping theorem and the existence problems for quasiconformal mappings were repeating, with rather slight modifications, the analytical methods of Vekua and his school or, at least, were essentially relying on these arguments. In many cases, embarassingly enough, these works contain only marginal, if any, references to the sources.

Douady's latest proof in [16] relies on the $L^{2}$ solution, expressed in terms of the Fourier transform (7) above, of the singular integral equation (4), (13) and on the 
solution (12) in the class $W_{\text {loc }}^{1,2}$ of the Beltrami equation (1). However, instead of the $L^{p}, p>2$, a priori estimates as above, he returns to the Grötzsch-LavrentievMorrey-Ahlfors results ${ }^{3}$ on the uniform Hölder estimates for QC-maps - reappearing in all compactness arguments of the elliptic theory of p.d.e.'s and quasiconformal mappings with bounded dilatation - and proposes a rather long way to go, with many references to exterior results, before achieving the existence theorem. See also the comments of Kra \& Earle in the second edition of [1].

In contrast, the deep theory of Lavrentiev [20], [21] and his followers: Volkovyskii [34], Belinskii [3], Pesin [26], using mainly direct geometric methods, contains many far reaching new ideas, so far only partially exploited and waiting, see [12], [27], for a modern, up-to-date presentation.

We leave aside the extensively growing and important research on mappings of finite distortion and their various generalizations.

The work of Vekua and his school on the solutions of the Beltrami equation yielded much more than the previous methods due to Lichtenstein [24], Lavrentiev [20] or Morrey [25], where, in various forms, the Riemann mapping theorem for QC-maps was proved.

The explicit representation formulas of Vekua's school and related a priori estimates for global mapping problems, created a powerful and flexible tool and a method to attack many local and global problems, inaccessible in any preceding theory. The study of quasiconformal extensions of holomorphic univalent functions and of the theory of deformations of planar quasiconformal mappings is hardly conceivable without these tools. They serve as a solid foundation for the development of important applications of the theory inside as well as outside the planar elliptic p.d.e. theory. The long list of the first ones starts with the deep results of Vinogradov and Danilyuk on basic boundary value problems for general elliptic equations and generalized analytic functions described in Vekua's monograph [33]. For the latter, i.e. applications outside the GAF, it is enough to mention the deep and beautiful ideas and constructions of the Ahlfors-Bers school in the theory of Teichmüller spaces, moduli spaces and Kleinian groups or the results in complex holomorphic dynamics [18], [1] (the 2006 edition).

It is necessary to stress here that the explicit formulas (12) and (23) written in the form

$$
\begin{aligned}
w_{\bar{z}} & =\omega=(1-q S)^{-1} q, \\
\text { and } \quad w_{z}-1 & =S \omega=S(1-q S)^{-1} q
\end{aligned}
$$

show that the derivatives $w_{\bar{z}}$ and $w_{z}$ of the principal solution (12) depend holomorphically, in the general functional sense, on the complex dilatation $q$. This functional dependence, naturally, implies that, if the dilatation $q(z)$ itself depends on some parameters $t$, holomorphically, real analytically, smoothly or just continuously, then the principal solutions (12) depend holomorphically, smoothly... etc., as the case may be, on these parameters. We will give some more comments on this topic later.

In [33] the existence of homeomorphic solutions of the complex Beltrami equation is also discussed in the compactified complex plane $\widehat{\mathbf{C}}$, identified with the Riemann sphere $S^{2}$. In this case, for the general measurable dilatation satisfying the condition (2) only, the homeomorphic solution cannot be in general represented by formula

\footnotetext{
${ }^{3}$ All these authors seem to assume a priori continuity or even local homeomorphism of the mappings.
} 
(12). However, as shown in [33], the principal homeomorphism can be constructed by the composition of two homeomorphisms of type (12) obtained by splitting the complex dilatation $q(z)=q_{1}+q_{2}$ with $q_{1}(z)$ and $q_{2}(1 / z)$ compactly supported, and a simple natural change of variables.

Also the behaviour of the complex dilatation $q_{w} \equiv \frac{w_{\bar{z}}}{w_{z}}$ under composition of quasiconformal mappings $f=w \circ v^{-1}$ is discussed in [8] and the simple, but important, formula

$$
q_{f}=\left\{\frac{q_{w}-q_{v}}{1-\overline{q_{v}} q_{w}} \frac{v_{z}}{\overline{v_{z}}}\right\} \circ v^{-1}
$$

appears and is used, at some crucial points, in [8].

Let us return to comments on the proof of Lemma 3: Consider the convex set $\Sigma$ of mappings of the form (15) parametrized by the densities $\widetilde{\omega} \in L^{p}\left(\mathbf{C}_{w}\right)$ for some fixed admissible $p>2$. For $z=z(w) \in \Sigma$ consider the principal solution $\psi(w)$ of the conjugate linear Beltrami equation

$$
\frac{\partial \psi}{\partial \bar{w}}+\widetilde{q}(w) \frac{\overline{\partial \psi}}{\partial w}=0
$$

with $\widetilde{q}(w) \equiv q(z(w))$.

This defines the nonlinear map $\psi=F(z)$ of $\Sigma$ into $\Sigma$. Since (30) is again a Beltrami equation in the $w$-plane, with the same uniform ellipticity estimate as (1), Lemma 1 and Proposition 1 hold and a priori estimates follow. Hence $F$ is compact and the fixed point of $F$ is the required solution of the quasilinear equation (14). For many analogous arguments see [8], [9], [10].

It follows also from the above a priori estimates (Proposition 1) that the iteration process

$$
\frac{\partial z_{n+1}}{\partial \bar{w}}+q\left(z_{n}(w)\right) \frac{\overline{\partial z_{n+1}}}{\partial w}=0
$$

defined on the class of principal (quasiregular) solutions defines uniquely the compact sequence of mappings in $\Sigma$ converging in $W_{\text {loc }}^{1, p}\left(\mathbf{C}_{w}\right)$ to the required solution of the quasilinear equation (14).

$$
z_{n}(w) \rightarrow z(w)
$$

converge locally uniformly and weakly in $W_{\text {loc }}^{1, p}$ to the (unique) solution of the equation (14).

Summing up, we can state that, with the proof of Lemma 3 reduced to (31) and (32), our Lemmata 1-6 and Propositions 1-5 described above, together with the paper [6], give a complete, fully self-contained (i.e., not requiring references to any earlier analytical or geometrical results), detailed and thus "elementary" proof of the basic theorems on the existence and structural properties of solutions of the planar measurable Beltrami equation.

Additional comments. The concept of the principal solution of form (12) or its slight generalization

$$
w(z)=a z-\frac{1}{\pi} \int_{\mathbf{C}} \frac{\omega(\zeta)}{\zeta-z} d \sigma_{\zeta}, \quad a-\text { complex constant }
$$

is meaningful for the general Beltrami equation

$$
w_{\bar{z}}-q(z) w_{z}-q_{1}(z) \bar{w}_{z}=0
$$


with the uniform ellipticity condition

$$
|q(z)|+\left|q_{1}(z)\right| \leq q_{0}<1, \quad q_{0}-\text { const. }
$$

These equations correspond to Lavrentiev's quasiconformal mappings [20], [21], with "two pairs of characteristics" [34], [28], [8], [12], and in Vekua's school they have been considered from the outset [7], [8], [33], [27].

The infinitesimal geometric meaning of a differentiable transformation $w=w(z)$ at a point $z_{0}$ is defined by the linear tangent map

$$
D w(z)(\xi)=w_{z}\left(z_{0}\right) \xi+w_{\bar{z}}\left(z_{0}\right) \bar{\xi} .
$$

It transforms ellipses in the tangent plane at $z_{0}$ into ellipses in the tangent plane at the image point $w\left(z_{0}\right)$.

Ellipses centred at $z$ are defined up to a similarity transformation by the ratio $p \geq 1$ of their semiaxes and, if $p>1$, the angle $\theta \bmod \pi$ between majoraxis and the positive $z$-axis, and denoted by $\mathscr{E}(p, \theta ; z)$ or $\mathscr{E}_{h}(p, \theta ; z)$ where $h$ is the length of the minoraxis. The pair $(p, \theta)$ is called the characteristic of the infinitesimal ellipse, and the family $\mathscr{E}_{h}(p, \theta ; z), h>0, z \in G$, is a field of infinitesimal ellipses (Lavrentiev field). A homeomorphism $w=w(z)$ is said to map the infinitesimal ellipse $\mathscr{E}(p, \theta ; z)$ onto $\mathscr{E}\left(p_{1}, \theta_{1} ; w(z)\right)$ if the tangent map $D w(z)$ transforms $\mathscr{E}(p, \theta ; z)$ onto $\mathscr{E}\left(p_{1}, \theta_{1} ; w(z)\right)$.

Analytically this is described in terms of the components $w_{\bar{z}}$ and $w_{z}$ in the tangent map Dw (36) by the general Beltrami equation (34) where the coefficients $q$ and $q_{1}$ are determined by the invertible formulas

$$
q(z)=-\frac{p-p^{-1}}{p+p^{-1}+p_{1}+p_{1}^{-1}} e^{2 i \theta}, \quad q_{1}(z)=\frac{p_{1}-p_{1}^{-1}}{p+p^{-1}+p_{1}+p_{1}^{-1}} e^{2 i \theta_{1}} .
$$

In particular the solutions of the Beltrami equation $(1)\left(q_{1} \equiv 0\right)$ map the field of ellipses $\mathscr{E}(p, \theta ; z)$ into infinitesimal circles $\left(p_{1} \equiv 1\right)$ whereas the conjugate Beltrami equations (14), (17) map the infinitesimal discs $(p \equiv 1)$ into ellipses $\left(p_{1} \geq 1\right)$.

We should stress that the clu of the Lavrentiev idea is that the "source" characteristic $(p, \theta)$ is mapped into the "target" characteristic $\left(p_{1}, \theta_{1}\right)$ independently of the considered particular solution of the general Beltrami equation (34) as long as the relation source $z$-target $w=w(z)$ is preserved. The formulae (37) describe then a pair of "distinguished" or canonical Lavrentiev fields intrinsic for the Beltrami system considered and a selected, pointwise correspondence $w=w(z)$. Of course any chosen solution $w=w(z)$ at every differentiability point trannsforms an arbitrary Lavrentiev field of infinitesimal ellipses into "some" Lavrentiev field whose characteristics $p_{1}, \theta_{1}$ at the image point $w(z)$ depend on the behaviour of the map at neighbouring points, i.e., on the values of the derivatives $w_{z}, w_{\bar{z}}$ at $z$.

Lavrentiev in his seminal paper of 1935 [20] defined QC-mappings as homeomorphic mappings of the unit disc $D$ onto itself such that at every point $z \in D$ the infinitesimal ellipse $\mathscr{E}(p(z), \theta(z) ; z)$ is mapped onto an infinitesimal circle in the sense defined above. He also proved the existence theorem for such mappings by a direct geometric construction without referring to any classical solutions of boundary value problems, e.g. in the Lichtenstein paper [24]. Thus Lavrentiev is probably to be credited for the first direct, self-contained proof of the global Riemann mapping theorem for a rather general class of complex Beltrami equations with continuous coefficients. 
The density $\omega(\zeta)$ of the principal solution (33) satisfies the singular integral equation

$$
\omega-q S \omega-q_{1} \bar{S} \omega=a q+\bar{a} q_{1},
$$

which is uniquely solvable and its $L^{2}$ solutions are necessarily in $L^{p}$ for some $p>2$. Equations of type (38) are linear over the real field only and were widely applied in [7], [8], [33] and many later works.

We state here a direct corollary of the above theory of Beltrami equation (1) which we formulate as

Proposition 6. The equation (34) has always a unique principal solution of the form (33). If $a \neq 0$ then the principal solution realizes a homeomorphic quasiconformal mapping of the full complex plane $\widehat{\mathbf{C}}$.

For $a=0$ the principal solution is identically $\equiv 0$ (Liouville theorem).

We stress the fact that, after the theory of Beltrami equations (1) is available as formulated above, no work at all is needed to prove Proposition 6. However, there would be a long way, though that is possible, see [10], before one could construct an analytical inverse mapping to (33) with the help of global solutions of some quasilinear general Beltrami equations (of type (14)). See also [10].

An important class of Beltrami equations (34) appears when the identity mapping $w(z)=z$ is a solution of $(34)$. These are characterized by the formula

$$
q(z)+q_{1}(z) \equiv 0
$$

and already appeared in [34] and [8]. In view of (37) the relation (39) reduces to the formulas $p \equiv p_{1}$ and $\theta \equiv \theta_{1}$ and have the beautiful geometric characterization in terms of Lavrentiev characteristics (Lavrentiev fields), see [34], [8] and [12]. In terms of the linear tangent map $D w$ of the given pointwise mappings $w=w(z)$ the Lavrentiev field (see [12]) associated with system (34) at point $z$ (in $T_{z} \mathbf{C}$ ) is parallel translated to the tangent plane $T_{w}$ at the image point $w=w(z)$. In [8] the systems (34)-(39) appeared in connection with the uniqueness problem for Riemann mapping corresponding to the general Beltrami system (34). The study of principal solutions (33) as a function of the parameter $a$ in this formula is an interesting topic and should be continued.

The study of the genuinely nonlinear Beltrami equations

$$
w_{\bar{z}}=H\left(z, w, w_{z}\right)
$$

for some complex valued function $H(z, w, \xi)$ has also been started by the author [10] in connection with the programme of introducing complex analytic methods to the Lavrentiev theory of fully nonlinear (implicit) first order systems (e.g. [21] and many other papers). Lavrentiev's geometric ellipticity concept was interpreted as the Lipschitz condition

$$
\left|H\left(z, w, \zeta_{1}\right)-H\left(z, w, \zeta_{2}\right)\right| \leq q_{0}\left|\zeta_{1}-\zeta_{2}\right|
$$

with $q_{0}=$ const. $<1$. Global existence and structure theorems for solutions of principal type (12) were proved, see [10] and [14].

As is well known, the principal solution (12) generates all solutions of the Beltrami equations (1). This is described in the famous so called Stoilow factorisation theorems [29]. This fact is crucial in establishing important structural properties of quasiconformal mappings, like homotopy, factorization into mappings with arbitrary small 
dilatations, differentiability (though the obtained differentiability results are far from the subtle results of Menshov (1931) on the differentiability of open mappings), etc., etc., see [8], [33], [23]. It gives also the parametrization of planar QC-mappings by $L^{p}$ solutions of the integral equation (13) (by densities in $L^{p}$, which also allows us to introduce the Banach manifold structure into the set of all QC-maps).

Formulas (12) and other related formulas give us a convenient tool to study the dependence of quasiconformal mappings $f(t, z)$ on parameters $t \in P, P$ denoting some parameter space. In the simplest case the parameter $t$ may vary in some interval of the real line. Representation formulas (12) allow us to reduce the problem to the study of parameter families of the corresponding complex "densities" $\omega(t) \equiv \omega(t, z) \equiv$ $\frac{\partial f(t, z)}{\partial \bar{z}}$ in their behaviour under small variations of the parameter, differentiation, etc.

The parameter derivatives $\dot{\omega}, \dot{q}, \dot{h}$ are then the partials $\dot{\omega}=\frac{\partial \omega}{\partial t}$ etc., and are seen to be the solutions of the integral equation

$$
\dot{\omega}(t)-q(t) S(\dot{\omega})=\dot{q} \cdot S \omega+\dot{h},
$$

obtained from the integral equation (4) by "differentiation" with respect to the parameter $t \in P$. Naturally, it is assumed that the coefficient $q$ and the right hand side $h$ in equation (4) are differentiable with respect to the parameter $t$ (see [11], [13]).

If the parameter space is, e.g., an open subset of $\mathbf{R}^{n}$ or $\mathbf{C}^{n}$, the partials $\frac{\partial}{\partial t}$ may be replaced by some "total" Fréchet type differential operators, in general denoted by the symbol $D_{t}$. Then the equation (42) takes the "general" form

$$
\dot{\omega}(t)-q(t) S(\dot{\omega})=D_{t} q \cdot S \omega+D_{t} h .
$$

In the literature there are many examples of this type of studies [2], [11], [13]. They all rely on the a priori estimates of the solutions of integral equations of type (4), which are, essentially, consequences of our assumptions (10).

In the particular case when $t$ is the complex structure parameter and $D_{t} \equiv \frac{\partial}{\partial \bar{t}}$ (42) together with the assumption $\frac{\partial q}{\partial t}=\frac{\partial h}{\partial \bar{t}}=0$ leads to the equation

$$
\frac{\partial \omega}{\partial \bar{t}} \equiv 0
$$

which implies $\frac{\partial f}{\partial \bar{t}} \equiv 0$ and is interpreted as holomorphic dependence of QC-maps on holomorphic parameters.

Note that the complex conjugate Beltrami equations (14) and (16) are linear only over the reals and the general differentiations $D_{t}$ should take this into account. This refers in particular to equations (31), (38) and implies that even for holomorphic in $t$ complex dilatations $q(t, z)$, the inverse mappings $f^{-1}(t, z)$ are holomorphic in $\bar{t}$ instead of $t$.

For normalized quasiconformal mappings of the complex plane, the unit disc and other "model" domains, explicit formulas of the type (12) allow us to calculate the Gateaux differential of the normalized mappings in their dependence on the infinitesimal variation of the complex dilatation (see [1], [13], and many other papers by Gutlyanskii), revealing the connections of the analytic theory of Beltrami equations with the study of deformations of conformal structures in the geometric function theory of Goluzin and Kufarev.

Some historical remarks scattered in this paper do not pretend to give, in any sense, a full and satisfactory account of the history of research in the area. It is also clear that in any sufficiently rich and mature mathematical theory progress is, 
generally speaking, the result of the collective effort of many researchers throughout the years. However, some landmarks can, and perhaps, even should be highlighted and it is proper and useful that this be done responsibly. I am convinced that the Beltrami equations and their applications lack a serious historical presentation though they certainly deserve one, with a view into the past as well as the future.

The idea of this paper was conceived when I visited the Mathematical Department of the Helsinki University, the Helsinki Technical University and the Jyväskylä University in April of the year 2009. I express sincere thanks to these Mathematical Departments. The main concepts and the proof of Proposition 5 were discussed with the leading specialists on the Beltrami equation and on quasiconformal mappings at these institutions.

At last let me also express my gratitude to the unknown referee for his not easy work to go through my awkward writing and the valuable remarks offered in his report.

\section{References}

[1] Ahlfors, L.: Lectures on quasiconformal mappings. - Van Nostrand, Princeton 1966; Second Edition, Univ. Lect. Series 38, Amer. Math. Soc., Providence, 2006.

[2] Ahlfors, L., and L. Bers: Riemann's mapping theorem for variable metrics. - Ann. of Math. (2) $72,1960,385-404$.

[3] Belinskil̆, P. P.: General properties of quasiconformal mappings. - Nauka, Novosibirsk, 1974 (in Russian).

[4] Bojarski, B.: Boundary value problems for elliptic systems with two independent variables. - Ph.D. dissertation and report, MGU, Moscow, 1954 (in Russian).

[5] Bojarski, B.: On a boundary value problem for a system of elliptic equations. - Dokl. Akad. Nauk SSSR (N.S.) 102, 1955, 201-204 (in Russian).

[6] Bojarski, B.: Homeomorphic solutions of the Beltrami systems. - Dokl. Akad. Nauk SSSR (N.S.) 102, 1955, 661-664 (in Russian).

[7] Bojarski, B.: On solutions of elliptic systems in the plane. - Dokl. Akad. Nauk SSSR (N.S.) 102, 1955, 871-874 (in Russian).

[8] Bojarski, B.: Generalized solutions of a system of differential equations of first order and of elliptic type with discontinuous coefficients. - Mat. Sb. N.S. 43:85, 1957, 451-503 (in Russian); English transl. in Rep. Univ. Jyväskylä Dept. Math. Stat. 118, 2009, available at www.jyu.fi/ science/laitokset/maths/tutkimus/reports.

[9] Bojarski, B.: General properties of solutions of elliptic systems in the plane. - In: Issled. sovr. probl. teor. funktsiĭ kompl. peremen. (Moscow Conf. 1957), ed. I. A. Markushevich, Gosfizmatizdat, Moscow, 1960, 461-483 (in Russian); Transl. in Fonctions d'une variable complexe, Probl. contemporaines, Gauthier-Villars, Paris, 1962, 254-268.

[10] Bojarski, B.: Quasiconformal mappings and general structural properties of systems of non-linear equations elliptic in the sense of Lavrentiev. - In: Symposia Mathematica XVIII (Convegno sulle Trasformazioni Quasiconformi e Questioni Connesse, INDAM, Rome, 1974), Academic Press, London, 1976, 485-499.

[11] Bojarski, B.: Old and new on Beltrami equation. - In: Functional Analytic Methods in Complex Analysis and Applications to Partial Differential Equations (Trieste, 1988), World Scientific, River Edge, 1990.

[12] Bojarski, B.: Geometry of the general Beltrami equations. - In: Complex Analysis and Potential Theory, ed. T. Aliyev Azeroğlu and P. M. Tamrazov, World Scientific, Singapore, 2007, 66-83. 
[13] Bojarski, B., and V. Ya. Gutlyanskir: On the Beltrami equation. - In: Conference Proceedings and Lecture Notes on Analysis I (Tianjin, 1992), ed. Zhong Li, International Press, Cambridge, MA, 1994, 8-33.

[14] Bojarski, B., and T. Iwaniec: Quasiconformal mappings and non-linear elliptic equations I. - Bull. Pol. Acad. Sci. 27, 1974, 473-478.

[15] Calderón, A. P., and A. Zygmund: On the existence of certain singular integrals. - Acta Math. 88, 1952, 85-139.

[16] DouAdy, A.: Le théorème d'integrabilité des structures presque complexes (d'après des notes de X. Buff). - In: The Mandelbrot Set, Theme and Variations, Cambridge Univ. Press, Cambridge, 2000, 307-324.

[17] Hamilton, D. H.: Area distortion of quasiconformal mappings. - In: Handbook of Complex Analysis: Geometric Function Theory, vol. 1, Elsevier, Amsterdam, 2002, 147-160.

[18] Hubbard, J. H.: Teichmüller theory with applications to topology, geometry, and dynamics, vol. I: Teichmüller theory. - Matrix Edition, Ithaca, 2006.

[19] Kühnau, R. (ed.): Handbook of complex analysis: Geometric function theory, vol. 1-2. Elsevier, Amsterdam, 2002-2005.

[20] Lavrentiev, M. A.: Sur une classe de représentations continues. - Rec. Math. Moscou 42, 1935.

[21] Lavrentiev, M. A.: A fundamental theorem of the theory of quasiconformal mapping of plane regions. - Izv. Akad. Nauk SSSR 12, 1948 (in Russian).

[22] Lehto, O.: Univalent functions and Teichmüller spaces. - Springer, Berlin, 1987.

[23] Lehto, O., and K. I. Virtanen: Quasikonforme Abbildungen. - Grundlehren Math. Wiss. 126, Springer, Berlin, 1965; 2nd ed.: Quasiconformal mappings in the plane, Springer, Berlin, 1973.

[24] Lichtenstein, L.: Zur Theorie der konformen Abbildungen: Konforme Abbildungen nichtanalytischer singularitätenfreier Flächenstücke auf ebene Gebiete. - Bull. Acad. Sci. Cracovie, 1916, 192-217.

[25] Morrey, C. B.: On the solutions of quasi-linear elliptic partial differential equations. - Trans. Amer. Math. Soc. 43, 1938, 126-166.

[26] Pesin, I. N.: Metric properties of Q-quasiconformal mappings. - Mat. Sb. N.S. 40:82, 1956, 281-294.

[27] Renelt, H.: Elliptic systems and quasiconformal mappings. - John Wiley \& Sons, Chichester, 1988.

[28] Schapiro, Z.: Sur l'existence des représentations quasi-conformes. - Dokl. Akad. Nauk SSSR (N.S.) 30, 1941, 690-692.

[29] SтоїLow, S.: Brief summary of my research work. - In: Analysis and Topology, A volume dedicated to the memory of S. Stoïlow, World Scientific Publ., River Edge, NJ, 1998.

[30] VEKUA, I. N.: Systems of differential equations of the first order of elliptic type and boundary value problems, with an application to the theory of shells. - Mat. Sb. N.S. 31:73, 1952, 217-314.

[31] VEKUA, I. N.: The general representation of functions of two independent variables admitting a derivative in the sense of S. L. Sobolev. - Dokl. Akad. Nauk SSSR 89, 1953, 773-775 (in Russian).

[32] VEKUA, I. N.: The problem of reducing differential forms of elliptic type to canonical form and the generalized Cauchy-Riemann system. - Dokl. Akad. Nauk SSSR 100, 1955, 197-200 (in Russian).

[33] VekuA, I. N.: Obobshchennye analiticheskie funktsii. - Fizmatgiz, Moscow, 1959; English transl.: Generalized analytic functions. - Pergamon Press, Oxford, 1962.

[34] Volkovysky, L. I.: Quasiconformal mappings. - Lvov, 1954 (in Russian). 